\title{
SLC26A9-mediated chloride secretion prevents mucus obstruction in airway inflammation
}

\author{
Pinelopi Anagnostopoulou, ${ }^{1}$ Brigitte Riederer, ${ }^{2}$ Julia Duerr, ${ }^{1}$ Sven Michel, ${ }^{3}$ Aristea Binia, ${ }^{3}$ \\ Raman Agrawal, ${ }^{1}$ Xuemei Liu,2 Katrin Kalitzki, ${ }^{2}$ Fang Xiao,2 Mingmin Chen, ${ }^{2}$ \\ Jolanthe Schatterny, ${ }^{1}$ Dorothee Hartmann, ${ }^{4}$ Thomas Thum, ${ }^{4}$ Michael Kabesch, ${ }^{3}$ \\ Manoocher Soleimani, ${ }^{5}$ Ursula Seidler, ${ }^{2}$ and Marcus A. Mall1,6
}

\begin{abstract}
${ }^{1}$ Department of Translational Pulmonology, Translational Lung Research Center, Member of the German Center for Lung Research, University of Heidelberg, Heidelberg, Germany. 2Department of Gastroenterology, ${ }^{3}$ Department of Pediatric Pneumology, Allergy and Neonatology, Member of the German Center for Lung Research, and ${ }^{4}$ Institute of Molecular and Translational Therapeutic Strategies (IMTTS), Hannover Medical School, Hannover, Germany. ${ }^{5}$ Center on Genetics of Transport and Epithelial Biology, University of Cincinnati, Cincinnati, Ohio, USA.

${ }^{6}$ Division of Pediatric Pulmonology and Allergy and Cystic Fibrosis Center, Department of Pediatrics III, University of Heidelberg, Heidelberg, Germany.
\end{abstract}

\begin{abstract}
Asthma is a chronic condition with unknown pathogenesis, and recent evidence suggests that enhanced airway epithelial chloride $\left(\mathrm{Cl}^{-}\right)$secretion plays a role in the disease. However, the molecular mechanism underlying $\mathrm{Cl}^{-}$secretion and its relevance in asthma pathophysiology remain unknown. To determine the role of the solute carrier family 26, member 9 (SLC26A9) $\mathrm{Cl}^{-}$channel in asthma, we induced Th2-mediated inflammation via IL-13 treatment in wild-type and Slc26a9-deficient mice and compared the effects on airway ion transport, morphology, and mucus content. We found that IL-13 treatment increased $\mathrm{Cl}^{-}$secretion in the airways of wildtype but not Slc26a9-deficient mice. While IL-13-induced mucus overproduction was similar in both strains, treated Slc26a9-deficient mice exhibited airway mucus obstruction, which did not occur in wild-type controls. In a study involving healthy children and asthmatics, a polymorphism in the $3^{\prime}$ UTR of SLC26A9 that reduced protein expression in vitro was associated with asthma. Our data demonstrate that the SLC26 $\mathrm{Ag} \mathrm{Cl}^{-}$channel is activated in airway inflammation and suggest that SLC26A9-mediated $\mathrm{Cl}^{-}$secretion is essential for preventing airway obstruction in allergic airway disease. These results indicate that SLC26A9 may serve as a therapeutic target for airway diseases associated with mucus plugging.
\end{abstract}

\section{Introduction}

The clinical syndrome of asthma has evolved as one of the most common chronic diseases in children and adults, but its pathogenesis remains incompletely understood. Th2-mediated inflammation triggered by inhaled allergens plays an important role in mucus hypersecretion, airway remodeling, and airway hyperresponsiveness contributing to airflow limitation in asthma, and the airway epithelium is a central effector tissue in this process $(1,2)$. Recent studies demonstrated that Th2-dependent airway inflammation produces a pro-secretory airway epithelial ion transport phenotype characterized by upregulation of $\mathrm{Cl}^{-}$secretion and inhibition of $\mathrm{Na}^{+}$absorption in mouse airways, and have identified active modulation of airway ion transport as an additional epithelial response in the complex in vivo pathogenesis of asthma (3). Proper hydration of airway surfaces by coordinate regulation of absorption and secretion of ions and fluid by the airway epithelium plays a critical role in maintaining normal mucociliary clearance $(\mathrm{MCC})(4,5)$. Because mucin macromolecules are secreted in a condensed dehydrated form into the airway surface liquid (ASL), where they are hydrated to form mucin gels by binding of free water (6), we hypothesized that upregulation of fluid secretion may be required for maintenance of ASL homeostasis

Authorship note: Pinelopi Anagnostopoulou, Brigitte Riederer, and Julia Duerr contributed equally to this work. Marcus A. Mall and Ursula Seidler contributed equally as senior authors.

Conflict of interest: The authors have declared that no conflict of interest exists. Citation for this article: J Clin Invest. 2012;122(10):3629-3634. doi:10.1172/JCI60429. and prevention of mucus obstruction in the presence of mucin hypersecretion (3). However, expression of Cftr and Tmem16a, constituting the main molecular determinants of cAMP-regulated and $\mathrm{Ca}^{+}$-activated $\mathrm{Cl}^{-}$secretion in airway epithelia under physiological conditions (7), was not altered in mouse models of allergic asthma (3), and the molecular identity and pathophysiological relevance of the $\mathrm{Cl}^{-}$conductance underlying increased epithelial $\mathrm{Cl}^{-}$secretion in allergic airway disease remain unknown.

SLC26A9 (solute carrier family 26, member 9) is a member of the SLC26 family of anion transporters predominantly expressed in epithelia of the lung and upper GI tract $(8,9)$. Recent studies showed that the SLC26A9 protein functions as a $\mathrm{Cl}^{-}$channel with minimal $\mathrm{HCO}_{3}{ }^{-}$conductance that is regulated by CFTR and WNK kinases in heterologous cells (10-13). Further, SLC26A9 contributes to constitutive and cAMP-dependent $\mathrm{Cl}^{-}$secretion in human bronchial epithelial (HBE) cells, where it has been suggested to interact functionally with CFTR in vitro $(11,14)$. Based on its functional properties in transduced cells and expression pattern in human and mouse airways $(8,15)$, we hypothesized that SLC26A9 may function as an alternative $\mathrm{Cl}^{-}$channel that may contribute to ASL homeostasis in health and allergic airway disease. To test this hypothesis, we compared transepithelial ion transport in freshly excised bronchial tissues, lung morphology, and airway mucus content in wild-type versus Slc26a9-deficient (Slc26a9-/-) mice under physiological conditions and after intratracheal instillation of IL-13 to model Th2-mediated airway inflammation. Further, in a large human study population of 658 healthy children and 661 children with asthma, we tested whether polymorphisms in 
A

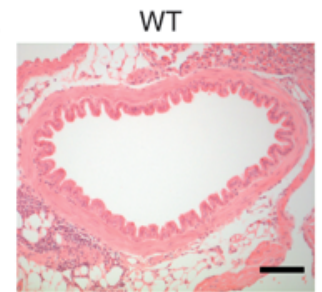

C

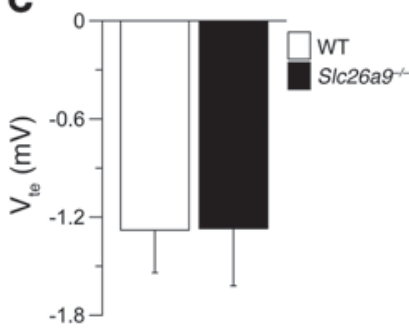

Slc26a9-

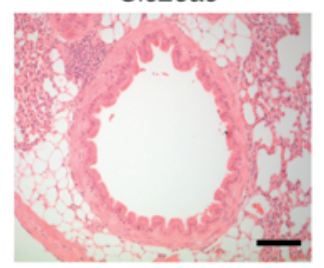

D

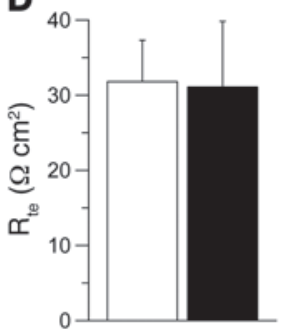

B

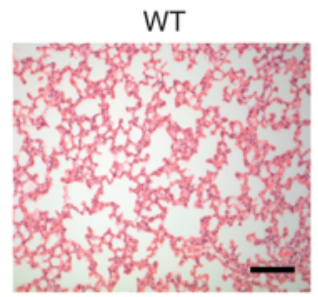

E

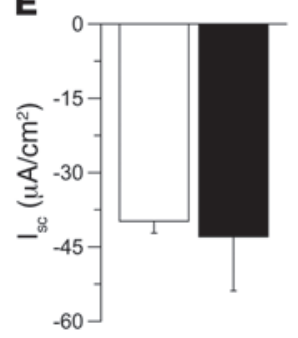

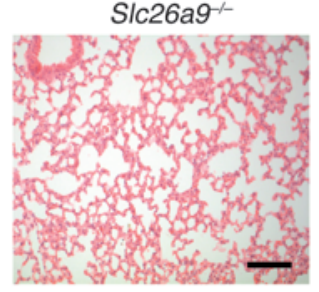

$\mathbf{F}$
G Amiloride- $\mathrm{CFTR}_{\text {inh }}-$ Bumetanide

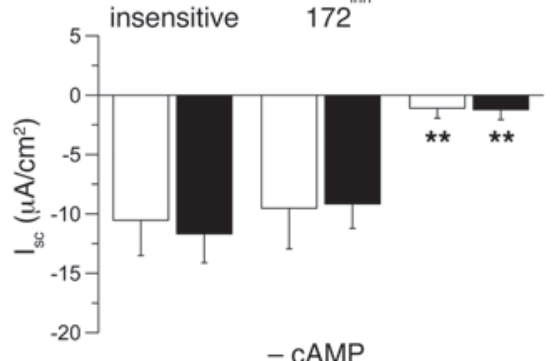

H Amiloride- $\mathrm{CFTR}_{\text {inh }}$ - Bumetanide insensitive

172

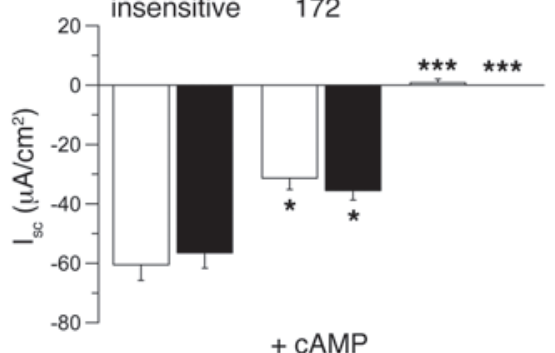

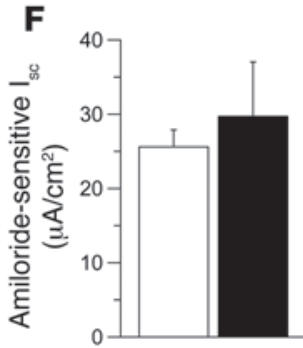

I

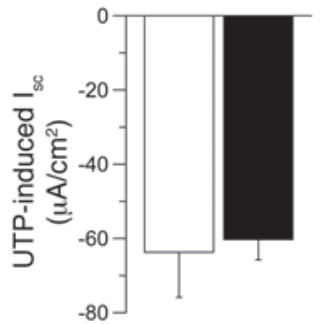

Figure 1

Lung morphology and airway ion transport are normal in naive S/c26a9-/- mice. (A and B) Representative morphology of conducting airways (A) and lung parenchyma (B) in naive wild-type and S/c26a9-/- mice. Sections were stained with H\&E. Scale bars: $100 \mu \mathrm{m}$. (C-F) Summary of $V_{\text {te }}(\mathbf{C})$, $R_{\mathrm{te}}(D)$, and $I_{\mathrm{sc}}(E)$ under basal conditions, and amiloride-sensitive $I_{\mathrm{sc}}(\mathbf{F})$ in freshly excised bronchial tissues from wild-type and S/c26a9-/- mice. $(\mathbf{G}$ and $\mathbf{H})$ Amiloride-insensitive $\mathrm{I}_{\mathrm{sc}}$ and effects of CFTR $\mathrm{inh}_{\mathrm{i}}-172$ and bumetanide in the absence (-cAMP) (G) and presence (+cAMP) (H) of cAMPmediated stimulation. (I) UTP-induced $I_{\mathrm{sc}}$ in the presence of amiloride and cAMP-dependent stimulation. $n=5-13$ mice per group. ${ }^{*} P<0.05$, ${ }^{\star \star} P<0.01,{ }^{* \star \star} P<0.001$ compared with mice of the same genotype.

the SLC26A9 gene are associated with asthma and determined the functional relevance of a select SNP (rs2282430) in the 3' UTR of SLC26A9 in luciferase gene reporter assays. Our studies demonstrate that SLC26A9-mediated $\mathrm{Cl}^{-}$secretion is activated and prevents airway mucus obstruction in allergic airway disease. These results suggest SLC26A9 as a potential disease modifier and novel therapeutic target in asthma and possibly other diseases caused by deficient ASL hydration, such as CF.

\section{Results and Discussion}

SLC26A9 does not contribute to airway $\mathrm{Cl}^{-}$secretion and is not essential for lung health under physiological conditions. We first compared lung morphology and ion transport properties in native bronchial tissues from naive wild-type and Slc26a9-/- mice. Consistent with previous immunolocalization studies $(8,15)$, Slc26a9 mRNA expression was detected by RT-PCR in bronchi from naive wild-type mice (Supplemental Figure 1; supplemental material available online with this article; doi:10.1172/JCI60429DS1). Morphological evaluation demonstrated that the conducting airways and alveolar airspaces were structurally normal and that lungs of Slc26a9-/- mice did not show evidence of spontaneous lung disease (Figure 1, A and B). To determine whether SLC26A9 contributed to constitutive $\mathrm{Cl}^{-}$ secretion in native airway epithelia, as previously described for cultured HBE cells (11), we first compared bioelectric properties of freshly excised bronchial tissues from wild-type and Slc26a9-/mice under $\mathrm{HCO}_{3}{ }^{-}$-free conditions. These analyses demonstrated that transepithelial voltage $\left(V_{t e}\right)$, transepithelial resistance $\left(R_{t e}\right)$, and short circuit current $\left(\mathrm{I}_{\mathrm{sc}}\right)$ were not different in wild-type versus Slc26a9-/- mice (Figure 1, C-E). Further, amiloride-sensitive $\mathrm{Na}^{+}$absorption mediated by epithelial $\mathrm{Na}^{+}$channels (ENaCs) and amiloride-insensitive $\mathrm{I}_{\mathrm{sc}}$ reflecting constitutive $\mathrm{Cl}^{-}$secretion in the presence of amiloride block (3) were normal in bronchial epithelia from Slc26a9 $9^{-1-}$ mice (Figure 1, F and G). The amiloride-insensitive $\mathrm{I}_{\mathrm{sc}}$ was not inhibited by $\mathrm{CFTR}_{\text {inh }^{-}}-172$, but was largely abolished by bumetanide in both wild-type and Slc26a9-/- mice (Figure 1G). Because previous studies showed that SLC26A9 stimulated cAMP/ PKA-regulated CFTR $\mathrm{Cl}^{-}$conductance in HBE cell lines (14), we next compared the magnitude of cAMP-induced $\mathrm{Cl}^{-}$secretion and effects of CFTR inh -172 in airways from wild-type and Slc26a9-/mice. In wild-type bronchi, cAMP-mediated stimulation induced a sustained $\mathrm{Cl}^{-}$secretory response that was significantly inhibited by CFTR $_{\text {inh }}-172$ (Figure $\left.1 \mathrm{H}\right)$. In contrast to previous results in cultured HBE cells transduced with SLC26A9 (14), our studies in native bronchial epithelia detected no differences in either cAMPinduced $\mathrm{I}_{\mathrm{sc}}$ or inhibition by $\mathrm{CFTR}_{\text {inh }}-172$ in tissues from Slc26a9-/compared with wild-type mice (Figure $1 \mathrm{H}$ ). The $\mathrm{CFTR}_{\text {inh }^{-}} 172-$ 
A

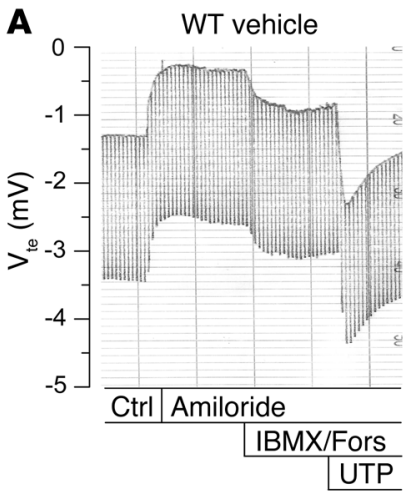

B

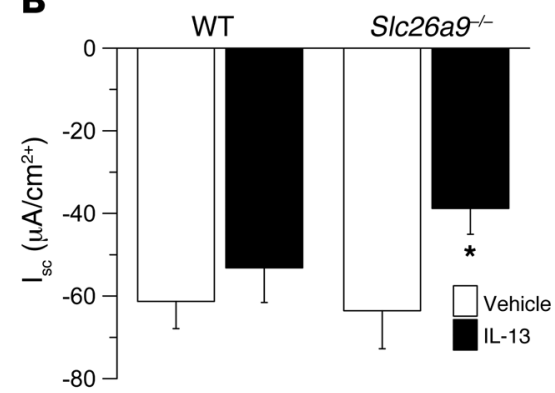

E

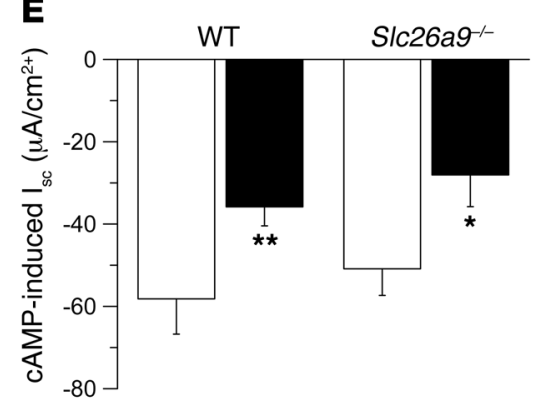

WT IL-13

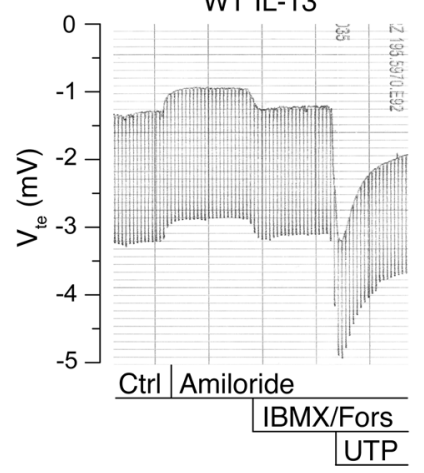

C

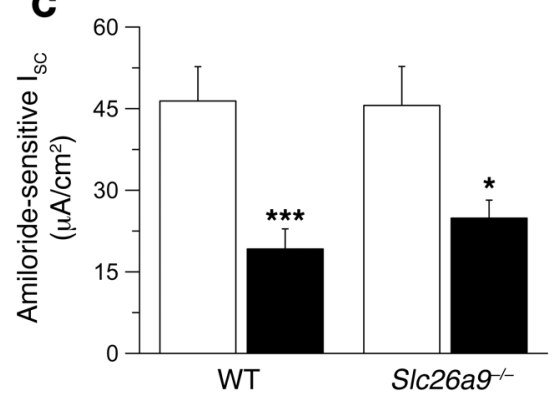

F

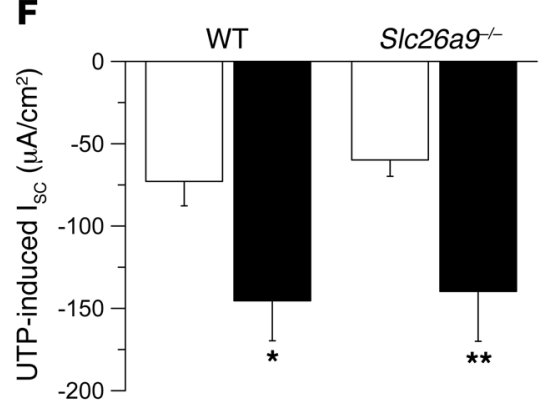

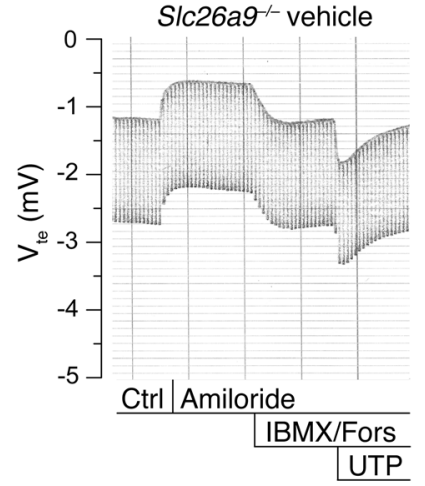

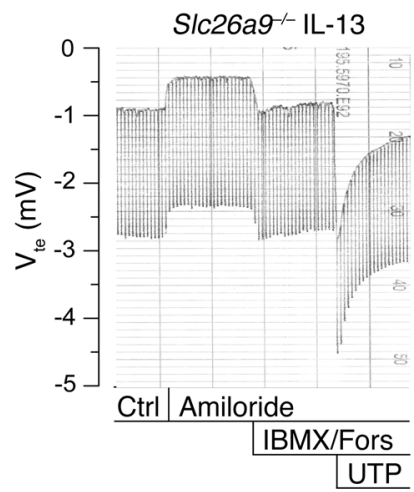

D
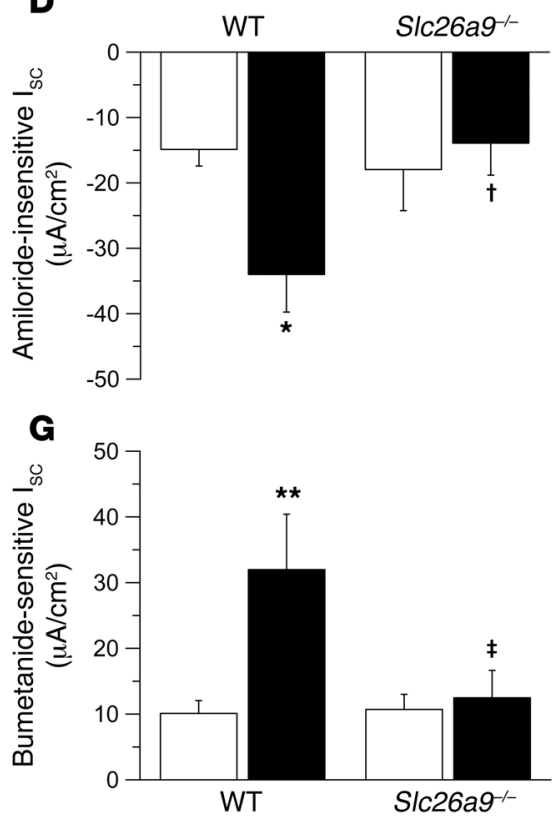

Figure 2

SLC26A9 determines constitutive $\mathrm{Cl}^{-}$secretion induced in Th2-mediated airway inflammation. (A) Original recordings of effects of amiloride, cAMPmediated (IBMX/forskolin [Fors]) activation, and UTP on $\mathrm{V}_{\text {te }}$ and $\mathrm{R}_{\mathrm{te}}$ across freshly excised bronchial tissues from vehicle- and IL-13-treated wildtype and S/c26a9-/- mice. Ctrl, control. (B-F) Summary of basal $I_{s c}(B)$, amiloride-sensitive $I_{s c}(\mathbf{C})$, amiloride-insensitive $I_{s c}(D)$, cAMP-induced $I_{s c}$ $(\mathrm{E})$, and UTP-induced $\mathrm{Isc}_{\mathrm{sc}}(\mathbf{F})$ in bronchi from vehicle-treated and IL-13-treated wild-type and S/c26a9-/- mice. (G) Summary of bumetanide-sensitive $\mathrm{I}_{\mathrm{sc}}$ in amiloride-pretreated tissues from vehicle- and IL-13-treated wild-type and S/c26a9-/- mice (G). $n=8-11$ mice per group. ${ }^{*} P<0.05$, ${ }^{* \star} P<0.01$, ${ }^{* * *} P<0.001$ compared with vehicle-treated mice of the same genotype. ${ }^{t} P<0.05, \ddagger P<0.01$ compared with IL-13-treated wild-type mice.

insensitive $\mathrm{I}_{\mathrm{sc}}$ was completely inhibited by bumetanide in tissues from both wild-type and Slc26a9-/- mice (Figure $1 \mathrm{H})$. To determine, whether SLC26A9-mediated $\mathrm{Cl}^{-}$transport and functional interaction with CFTR required the presence of $\mathrm{HCO}_{3}^{-}(10,11,14)$, we compared bioelectric properties in wild-type and Slc26a9-/- bronchi in the absence and presence of $\mathrm{HCO}_{3}{ }^{-}$. $\mathrm{HCO}_{3}{ }^{-}$had no effect on basal $\mathrm{I}_{\mathrm{sc}}$, constitutive $\mathrm{Cl}^{-}$secretion (amiloride-insensitive $\mathrm{I}_{\mathrm{sc}}$ ), or cAMP-induced $\mathrm{Cl}^{-}$secretion, and, as in $\mathrm{HCO}_{3}^{-}$-free conditions, constitutive and cAMP-dependent $\mathrm{Cl}^{-}$secretion were not different in wild-type compared with Slc26a9-/- tissues (Supplemental Figure 2). This lack of functional interaction between SLC26A9 and CFTR in native mouse airway epithelia may reflect species-dependent differences or differences in regulation of SLC26A9 and CFTR function in native airway epithelia versus transduced cell lines (14). Finally, we tested a potential contribution of SLC26A9 to $\mathrm{Ca}^{2+}$-activated $\mathrm{Cl}^{-}$conductance $(\mathrm{CaCC})$ by comparing UTP- induced $\mathrm{Cl}^{-}$secretory responses in bronchi from wild-type and Slc26a9 $9^{-/-}$mice. As expected, $\mathrm{Ca}^{2+}$-dependent activation induced a transient $\mathrm{Cl}^{-}$secretory response that was not different in wild-type versus Slc26a9-/- bronchi (Figure 1I). Taken together, these results argue against a role of SLC26 $99 \mathrm{Cl}^{-}$channels in constitutive or agonist-induced (cAMP-mediated and $\mathrm{Ca}^{2+}$-activated) $\mathrm{Cl}^{-}$secretion in native airway tissues and indicate that SLC26A9 function is not essential for lung health under physiological conditions in vivo.

SLC26A9 constitutes an epithelial $\mathrm{Cl}^{-}$conductance induced in Th2-mediated airway inflammation. Our previous studies demonstrated that Th2-mediated airway inflammation induces a substantial ( 2- to 3 -fold) increase in constitutive and $\mathrm{Ca}^{2+}$-activated $\mathrm{Cl}^{-}$secretion without changing expression levels of CFTR or TMEM16A in mouse airways (3). To test the hypothesis that SLC26 $99 \mathrm{Cl}^{-}$channels may be activated and contribute to enhanced $\mathrm{Cl}^{-}$secretion in allergic airway disease, we induced inflammation by intratra- 
A WT vehicle

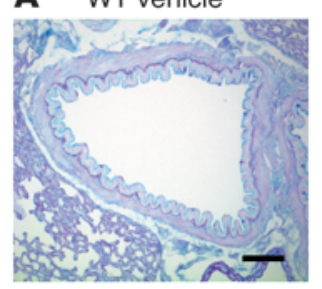

B

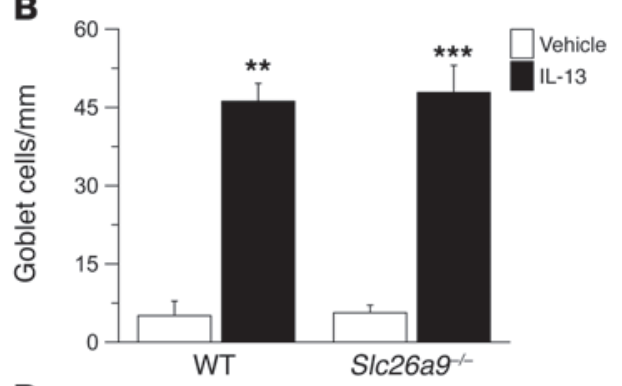

$$
\text { D }
$$

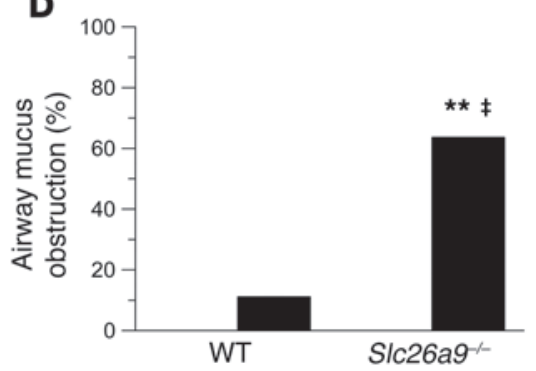

WT IL-13

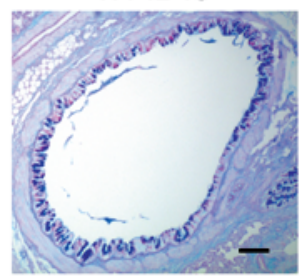

.
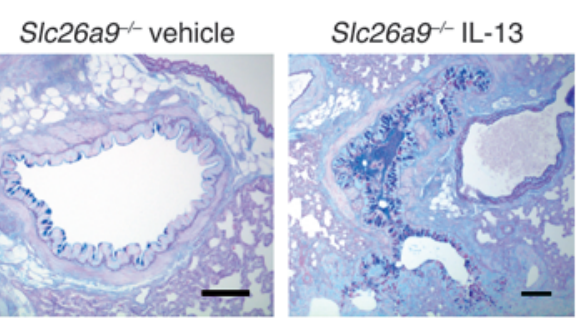

C

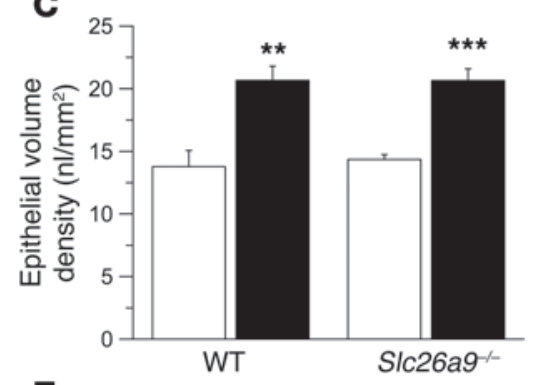

E

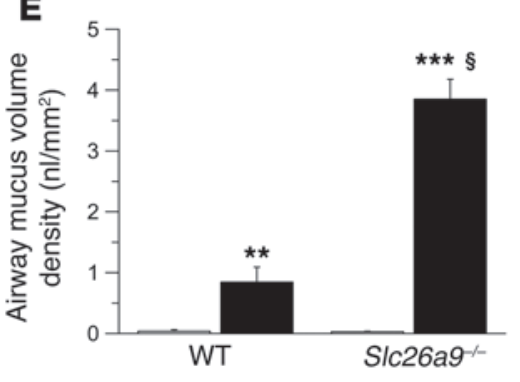

\section{Figure 3}

SLC26A9 prevents airway mucus obstruction in the presence of mucin overproduction in Th2-mediated airway disease. (A) Representative airway morphology in vehicleand IL-13-treated wild-type and Slc26a9-/- mice. Sections were stained with Alcian blue-PAS to determine the presence of goblet cells and airway mucus. Scale bars: $100 \mu \mathrm{m}$. (B-E) Summary of goblet cell densities (B) and epithelial volume density (C) in main axial airways; fraction (\%) of mice showing accumulation of airway mucus (D); and mucus volume density in the airway lumen (E) from vehicle-treated and IL-13-treated wild-type and S/c26a9-/- mice. $n=7-11$ mice for each group. ${ }^{* \star} P<0.01$, ${ }^{* \star \star} P<0.001$ compared with vehicletreated mice of the same genotype. $\ddagger P<0.01, \S P<0.001$ compared with IL-13-treated wild-type mice. cheal application of the key Th2 signaling molecule IL-13 (1) and compared effects on airway ion transport in wild-type and Slc26a9-/- mice (Figure 2). Treatment with IL-13 had no effect on $\mathrm{R}_{\mathrm{te}}$ in freshly excised bronchial tissues from wild-type and Slc26a9 $9^{-/}$mice (data not shown). In wild-type bronchi, IL-13 had no effect on basal $\mathrm{I}_{\mathrm{sc}}$, but reduced $\mathrm{ENaC}$-mediated $\mathrm{Na}^{+}$absorption and produced a significant increase in amiloride-insensitive $\mathrm{I}_{\mathrm{sc}}$ as well as $\mathrm{Ca}^{2+}$-activated $\mathrm{Cl}^{-}$secretion, as expected (ref. 3 and Figure 2, A-F). In bronchi from Slc26a9 $9^{-/}$mice, IL-13 treatment resulted in significantly reduced basal $\mathrm{I}_{\mathrm{sc}}$ compared with tissues from vehicle-treated Slc26a9 $9^{-/-}$mice and wild-type mice of both treatment groups (Figure 2, A and B). Inhibition of basal $\mathrm{I}_{\mathrm{sc}}$ was not due to differences in $\mathrm{ENaC}$-mediated $\mathrm{Na}^{+}$absorption, because IL-13 treatment reduced amiloride-sensitive ENaC currents to similar levels in bronchi from Slc26a9-/- and wild-type mice (Figure 2C). However, in contrast to wild-type tissues, bronchi from IL-13-treated Slc26a9 $9^{-1-}$ mice failed to exhibit increased amiloride-insensitive $\mathrm{I}_{\mathrm{sc}}$ (Figure 2D). As in bronchi from naive mice (Figure 1G), the amiloride-insensitive $\mathrm{I}_{\mathrm{sc}}$ was completely inhibited by bumetanide in tissues from vehicle- and IL-13-treated wild-type and Slc26a9-/mice (data not shown). Of note, the bumetanide-sensitive $I_{s c}$ was significantly increased by IL-13 in wild-type tissues, but remained unchanged in tissues from Slc26a9-/- mice (Figure 2G). Effects of IL-13 on ion transport in wild-type and Slc26a9 ${ }^{-/-}$bronchi were not different when tissues were studied in the absence or presence of $\mathrm{HCO}_{3}{ }^{-}$(Supplemental Figure 2). A similar bioelectric pattern with abrogation of IL-13-induced constitutive $\mathrm{Cl}^{-}$conductance was also observed in native tracheal tissue from Slc26a9-/- mice (data not shown), demonstrating that Th2-mediated inflammation induced SLC26A9-dependent $\mathrm{Cl}^{-}$secretion in different regions of the conducting airways. In contrast to effects on constitutive $\mathrm{Cl}^{-}$ secretion, IL-13-induced upregulation of CaCC activity did not differ in Slc26a9 $9^{-1-}$ compared with wild-type airways (Figure 2F).

Taken together, these results demonstrate that SLC26A9 constitutes the $\mathrm{Cl}^{-}$channel responsible for increased constitutive $\mathrm{Cl}^{-}$secretion in Th2-mediated inflammation, and link SLC26A9 function to allergic airway disease. On the other hand, our data indicate that SLC26A9 is not implicated in Th2-mediated upregulation of $\mathrm{Ca}^{2+}$-activated $\mathrm{Cl}^{-}$secretion (Figure $2 \mathrm{G}$ ). Of note, we previously showed that upregulation of $\mathrm{Ca}^{2+}$-activated $\mathrm{Cl}^{-}$ secretion in allergic airway disease is mediated by STAT6 signaling, whereas the increase in constitutive $\mathrm{Cl}^{-}$secretion is STAT6 independent (3), indicating that SLC26A9 and CaCC are regulated by distinct mechanisms. In our present study, expression analyses by semi-quantitative real-time RT-PCR demonstrated that increased SLC26A9 $\mathrm{Cl}^{-}$channel function did not correlate with transcript levels in native airway tissues from IL-13-treated wild-type mice (Supplemental Figure 1), suggesting that SLC26A9 $\mathrm{Cl}^{-}$conductance was regulated on the post-transcriptional level. In this context, it is noteworthy that the activity of SLC26A9, similar to several other ion channels and transporters implicated in electrolyte homeostasis in $\mathrm{Cl}^{-}$-handling epithelia, is inhibited by WNK4 and other WNK kinases via inhibition of SLC26A9 protein expression at the cell surface (13). Given that WNK4 is expressed in the lung (16), we speculate that WNKs may inhibit SLC26A9 in airway epithelia under physiological conditions and act as a molecular switch to activate SLC26A9-mediated $\mathrm{Cl}^{-}$secretion in response to stimuli released during allergic airway inflammation. 
A

SLC26A9 3' UTR WT 5' AAA CAC AUG AGG CAG AGG G $3^{\prime}$ SLC26A9 3' UTR SNP 5' AAA CAC AUG AAG CAG AGG G 3' hsa-miR-632

B
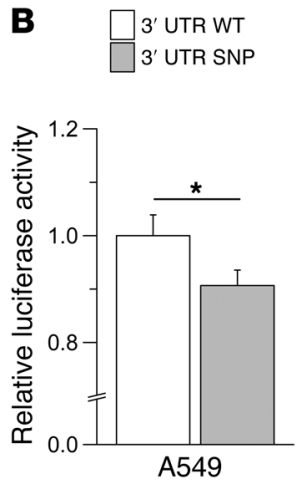
3' AGG GUG UCC UUC GUC UGU G $5^{\prime}$

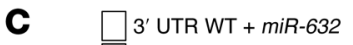
3' UTR SNP + miR-632

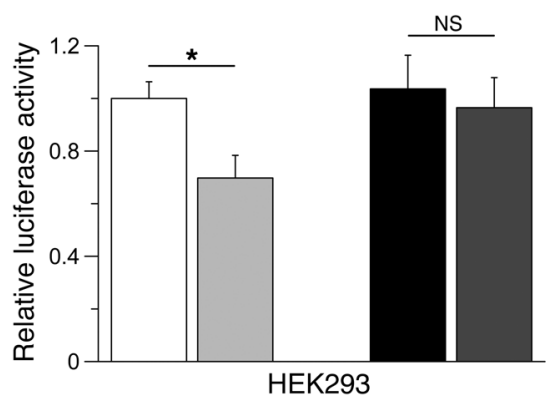

\section{Figure 4}

A SNP in the 3' UTR of SLC26A9 reduces reporter gene activity in luciferase reporter assays. (A) Alignment of the partial wild-type $3^{\prime}$ UTR sequence of SLC26A9 (nucleotides 4511-4529) with the SNP rs2282430 (indicated by arrowhead) and hsa-miR-632. (B) Summary of relative luciferase activity of reporter constructs containing the wildtype versus polymorphic (SNP) 3' UTR of SLC26A9 transfected into A549 cells. (C) Effects of co-transfection with precursor $h s a-m i R-632$ or a pre-miR negative control (miR-Ctrl) on luciferase activity of wild-type versus SNP SLC26A9 3' UTR constructs in HEK293 cells. $n=10-11$ experiments per group. ${ }^{\star} P<0.05$.
SLC26A9-mediated $\mathrm{Cl}^{-}$secretion prevents mucus obstruction in Th2mediated airway inflammation. To determine the functional consequences of SLC26A9-mediated $\mathrm{Cl}^{-}$secretion on mucus homeostasis in allergic airway disease, we next compared the airway mucus phenotype in naive and IL-13-treated wild-type and Slc26a9 $9^{-/-}$mice (Figure 3). Bronchi from IL-13-treated wild-type mice exhibited goblet cell metaplasia with increased epithelial mucin production and epithelial hypertrophy, as expected (Figure 3, A-C, Supplemental Figure 3, and ref. 3). In airways from Slc26a9-/- mice, IL-13 induced goblet cell metaplasia to levels similar to those in wildtype mice (Figure 3, A-C, and Supplemental Figure 3), indicating that SLC26A9 was not involved in epithelial remodeling and mucin overproduction caused by Th2-mediated inflammation. Consistent with previous studies in mouse models of asthma (4), accumulation of mucus in the airway lumen was rarely observed (Figure 3D), and morphometric analyses identified only small amounts of intraluminal mucus in airways from IL-13-treated wild-type mice (Figure 3E). In contrast, airway mucus obstruction was observed in the majority of IL-13-treated Slc26a9 $9^{-/-}$mice (Figure 3D). Further, airway morphometry demonstrated that airway mucus content was significantly increased in IL-13-treated Slc26a9-/- compared with wild-type mice (Figure 3E). When viewed in combination with the results from our bioelectric studies (Figure 2), these data demonstrate that SLC26A9-mediated $\mathrm{Cl}^{-}$secretion plays an essential role in preventing mucus obstruction in allergic airway disease.

Genetic variants in SLC26A9 are associated with reduced protein expression and increased risk for asthma. To investigate whether SLC26A9 may also play a role in human asthma, we analyzed 661 children with asthma and 658 healthy subjects by chip genotyping and imputation (17) for effects of genetic variants within the SLC26A9 locus on asthma risk (Supplemental Figure 4 and Supplemental Table 1). We identified several SNPs in the 3' UTR of SLC26A9 (rs12031234, rs2282429, rs2282430) that were associated with asthma in this population (highest odds ratio, 1.48; $95 \%$ confidence interval $[\mathrm{CI}], 1.1-2.0 ; P=0.012)$. In silico analyses predicted that the A allele of rs2282430 (SNP) strengthens binding of hsa-miR-632 to the SLC26A9 3' UTR (Figure 4A). In luciferase reporter assays in A549 cells, known to express SLC26A9 endogenously (8), luciferase activity was significantly reduced in cells transfected with the polymorphic compared with the wild-type 3' UTR $(P=0.02)$ (Figure 4B). Further, studies in HEK293 cells demonstrated that transfection of precursor $b s a-m i R-632$ reduced luciferase activity significantly in cells co-transfected with the SNP compared with the wild-type $3^{\prime}$ UTR $(P<0.05)$ (Figure 4C). Taken together, these results suggest that the SNP in the SLC26A9 3' UTR identified by our genetic analyses reduces protein expression, indicate that binding of hsa-miR-632 is involved in this process, and provide initial evidence that SLC26A9 may be involved in asthma pathogenesis in humans.

In summary, this study demonstrates that SLC26A9 constitutes a $\mathrm{Cl}^{-}$channel in native airway epithelia that is quiescent under physiological conditions but constitutively active to promote $\mathrm{Cl}^{-}$ secretion and hydration of airway surfaces in Th2-driven airway disease. To our knowledge, this is the first report showing direct involvement of an epithelial ion channel in the in vivo pathogenesis of asthma. The observation that genetic deletion of Slc26a9 produced significant airway mucus obstruction in allergic airway disease indicates that SLC26A9-mediated $\mathrm{Cl}^{-}$secretion is essential for maintaining ASL homeostasis required for effective MCC in the presence of mucus hypersecretion. These results also suggest SLC26A9 as a potential disease modifier and novel therapeutic target, especially in asthma phenotypes associated with substantial airway mucus obstruction, which remains difficult to treat, responds poorly to current anti-asthmatic drugs such as bronchodilators and antiinflammatory agents, and continues to contribute to asthma-related deaths (18). In this context, it is noteworthy that upregulation of SLC26A9-mediated $\mathrm{Cl}^{-}$secretion was independent of STAT6 signaling (3), suggesting that opportunities exist to activate SLC26A9 selectively without concomitant induction of STAT6-dependent airway pathologies such as eosinophilia, mucus hypersecretion, airway hyperresponsiveness, and airway remodeling (2). Our data also demonstrate that upregulation of transiently active $\mathrm{CaCC}$, in contrast to constitutively active SLC26A9, was not sufficient to prevent airway mucus obstruction in Th2-mediated airway disease. These results are consistent with observations in patients with $\mathrm{CF}$, in whom increased CaCCmediated $\mathrm{Cl}^{-}$secretion is not sufficient to compensate for deficient CFTR $\mathrm{Cl}^{-}$channel function and prevent dehydration-induced mucus plugging and airway disease (4). Therefore, as an alternative $\mathrm{Cl}^{-}$channel that mediates sustained $\mathrm{Cl}^{-}$secretion, SLC26A9 may also serve as a novel therapeutic target to counteract impaired epithelial $\mathrm{Cl}^{-}$secretion in $\mathrm{CF}$. 


\section{Methods}

Mice. Experiments were performed with 8- to 20-week-old Slc26a9-/- mice and wild-type littermates on the 129/SVJ background (9).

IL-13 instillation. IL-13 was instilled intratracheally as previously described (3) and detailed in Supplemental Methods.

Histology and airway morphometry. See Supplemental Methods for details.

Electrogenic ion transport measurements. See Supplemental Methods for details.

Real time RT-PCR. See Supplemental Methods for details.

Genetic association studies. Asthma patients from the Multicenter Asthma Genetics in Childhood (MAGIC) study were compared with randomly selected children from the cross-sectional International Study of Asthma and Allergies in Childhood, phase II (ISAAC II) study (17). A total of 1,319 subjects (661 with asthma) were analyzed in this study as described in Supplemental Methods.

Cloning of SLC26A9 3' UTR and luciferase gene reporter assays. See Supplemental Methods for details.

Statistics. Data were analyzed with SigmaStat version 3.1 (Systat Software) and are reported as mean \pm SEM. We performed statistical analyses using 2-tailed Student's $t$ test, Mann-Whitney rank-sum test, 1-way ANOVA, and Fisher exact test as appropriate, and $P<0.05$ was accepted to indicate statistical significance.

Study approval. All animal studies were approved by the local animal welfare authorities responsible for the Hannover Medical School (Regierungspräsidium Oldenburg, Niedersachsen) and the University of Heidelberg (Regierungspräsidium Karlsruhe, Baden-Württemberg). Protocols of the MAGIC and ISAAC studies (17) were approved by the ethics committees of the participating centers (University Children's Hospital, Ludwig Maximilian University, Munich; Department of Experimental Pneumology, Ruhr-University Bochum; University

1. Elias JA, Lee CG, Zheng T, Ma B, Homer RJ, Zhu $Z$. New insights into the pathogenesis of asthma. J Clin Invest. 2003;111(3):291-297.

2. Kuperman DA, et al. Direct effects of interleukin-13 on epithelial cells cause airway hyperreactivity and mucus overproduction in asthma. Nat Med. 2002; 8(8):885-889.

3. Anagnostopoulou P, Dai L, Schatterny J, Hirtz S, Duerr J, Mall MA. Allergic airway inflammation induces a pro-secretory epithelial ion transport phenotype in mice. Eur Respir J. 2010;36(6):1436-1447.

4. Mall MA. Role of cilia, mucus, and airway surface liquid in mucociliary dysfunction: lessons from mouse models. J Aerosol Med Pulm Drug Deliv. 2008; 21(1):13-24.

5. Mall M, Grubb BR, Harkema JR, O’Neal WK, Boucher RC. Increased airway epithelial $\mathrm{Na}^{+}$absorption produces cystic fibrosis-like lung disease in mice. Nat Med. 2004;10(5):487-493.

6. Verdugo P. Mucin exocytosis. Am Rev Respir Dis. 1991;

Children's Hospital, University of Cologne; Children's Department, Marien-Hospital, Wesel; University Children's Hospital, Albert Ludwig University, Freiburg; Children's Department, Feldkirch Hospital; University Children's Hospital Vienna), and written informed consent was obtained from the parents of all children.

\section{Acknowledgments}

This study was supported by grants from the Deutsche Forschungsgemeinschaft(MA 2081/3-3, MA 2081/4-1, SE 460/13-4, SE 460/9-6, and SFB621/C9) and Bundesministerium für Bildung und Forschung (DZL 82DZL00401, NGFN 01GS0810, and IFB-Tx 01EO0802). Genotyping of patients and controls was supported by EC contract 018996/GABRIEL. We thank Stephanie Hirtz, Brigitte Rausch, Regina Engelhardt, Natascha Cirpka, and Silke Thiele for expert technical assistance.

Received for publication August 9, 2011, and accepted in revised form July 12, 2012.

Address correspondence to: Marcus A. Mall, Department of Translational Pulmonology, Translational Lung Research Center, University of Heidelberg, Im Neuenheimer Feld 350, 69120 Heidelberg, Germany. Phone: 49.6221.568840; Fax: 49.6221.568806; E-mail: Marcus.Mall@med.uni-heidelberg.de. Or to: Ursula Seidler, Department of Gastroenterology, Hannover Medical School, Carl-Neuberg-Str. 1, 30625 Hannover, Germany. Phone: 49.511.5329427; Fax: 49.511.532.8428; E-mail: Seidler. Ursula@mh-hannover.de.

144(3 pt 2):S33-S37.

7. Rock JR, et al. Transmembrane protein 16A (TMEM16A) is a $\mathrm{Ca}^{2+}$-regulated $\mathrm{Cl}^{-}$secretory channel in mouse airways. J Biol Chem. 2009; 284(22):14875-14880.

8. Lohi $\mathrm{H}$, et al. Functional characterization of three novel tissue-specific anion exchangers SLC26A7, -A8, and -A9. J Biol Chem. 2002;277(16):14246-14254.

9. Xu J, et al. Deletion of the chloride transporter Slc26a9 causes loss of tubulovesicles in parietal cells and impairs acid secretion in the stomach. Proc Natl Acad Sci US A. 2008;105(46):17955-17960.

10. Loriol C, et al. Characterization of SLC26A9, facilitation of $\mathrm{Cl}^{-}$transport by bicarbonate. Cell Physiol Biochem. 2008;22(1-4):15-30.

11. Bertrand CA, Zhang R, Pilewski JM, Frizzell RA. SLC26A9 is a constitutively active, CFTR-regulated anion conductance in human bronchial epithelia. J Gen Physiol. 2009;133(4):421-438.

12. Chang $M H$, et al. Slc26a9 is inhibited by the
R-region of the cystic fibrosis transmembrane conductance regulator via the STAS domain. J Biol Chem. 2009;284(41):28306-18318.

13. Dorwart MR, Shcheynikov N, Wang Y, Stippec S, Muallem S. SLC26A9 is a $\mathrm{Cl}^{-}$channel regulated by the WNK kinases. J Physiol. 2007;584(pt 1):333-345.

14. Avella M, Loriol C, Boulukos K, Borgese F, Ehrenfeld J. SLC26A9 stimulates CFTR expression and function in human bronchial cell lines. J Cell Physiol. 2011;226(1):212-223.

15. Chang MH, et al. Slc26a9-anion exchanger, channel and $\mathrm{Na}^{+}$transporter. J Membr Biol. 2009; 228(3):125-140.

16. Kahle KT, et al. WNK4 regulates apical and basolateral $\mathrm{Cl}^{-}$flux in extrarenal epithelia. Proc Natl Acad Sci US A. 2004;101(7):2064-2069.

17. Michel $S$, et al. Unifying candidate gene and GWAS approaches in asthma. PLoS One. 2010;5(11):e13894.

18. Kuyper LM, et al. Characterization of airway plugging in fatal asthma. Am JMed. 2003;115(1):6-11. 